\title{
Improving the bioavailability of curcumin: is micro/nanoencapsulation the key?
}

\author{
Sabrina Barbosa de Souza Ferreira ${ }^{1}$ \& Marcos Luciano Bruschi ${ }^{*, 1}$ \\ ${ }^{1}$ Laboratory of Research \& Development of Drug Delivery Systems, Postgraduate Program in Pharmaceutical Sciences, Department \\ of Pharmacy, State University of Maringa, Maringa, Brazil \\ *Author for correspondence: mlbruschi@uem.br
}

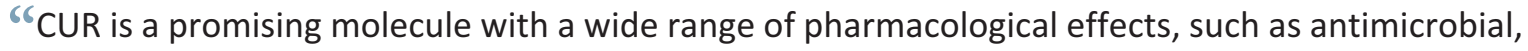 anti-inflammatory, anticancer and wound healing, as well as effects in chronic diseases, such as Alzheimer's disease and diabetes. However, its physicochemical properties are not favorable to the development of new pharmaceutical forms"

First draft submitted: 18 December 2018; Accepted for publication: 3 January 2019; Published online: 6 February 2019

Keywords: bioavailability • curcumin • nanotechnology • polyphenols • therapy • turmeric

During the last decades, substances from secondary metabolism of a number of plants and their derivatives have been investigated due to their important biological activities and advantages in pharmacological therapy. Among them, polyphenols compose a group of substances showing a variety of simple and complex chemical structures and can display multiple phenolic functional groups [1]. Curcumin (CUR), a yellow phytopolyphenol present in the rhizomes of turmeric (Curcuma longa Linn), has displayed multiple biological properties. This plant belongs to the ginger family (Zingiberaceae) being found in subtropical and tropical regions of India, China and Latin America. The dietary spice turmeric is extensively utilized in curries and mustards exhibiting $2-5 \%$ of curcuminoids: demethoxycurcumin, bisdemethoxycurcumin and cyclocurcumin [2-5].

Turmeric has been considered an important therapeutic agent in Chinese and Indian traditional medicine for supporting the blood, liver, joints, immune system and digestive tract [2,6]. CUR was isolated from turmeric, identified and purified almost two centuries ago by Vogel and Pelletier. In 1910, Milobedzka and Lampe identified its chemical structure. The biological properties of CUR were determined in the mid-twentieth century $[5,7]$.

CUR is safe even at high doses (12 g per day) in humans, but displays low aqueous solubility $(3.12 \mathrm{mg} / \mathrm{l}$ at $25^{\circ} \mathrm{C}$ ) [8], poor absorption, apparent rapid metabolism and rapid systemic elimination, resulting in problems of bioavailability [9]. Many approaches have been investigated to improve the CUR bioavailability, such as the use of adjuvant that interferes with glucuronidation, the use of structural analogs of CUR and also the use of technological strategies. Among them, the micro/nanoencapsulation has emerged as one of the most investigated strategies.

\section{Pharmacological properties}

CUR is considered as a pleiotropic molecule and multiple biological effects have been reported, such as the antimicrobial, anti-inflammatory, antioxidant, anticancer and some effects against diabetes $[6,10]$. Its antioxidant activity is due to the phenolic functional group with hydrogen that possesses donating ability [10]. Moreover, some articles have reported the action of CUR in Alzheimer's disease by inhibiting and reduction A $\beta$ oligomers and lowering cell cytotoxicity, suppression of inflammation and oxidative damage, as well as, improving cognitive deficits [11].

CUR also exerts potential activity in metabolic diseases such as diabetes by increasing of insulin sensitivity as well as hypoglycemic and hypocholesterolemic effects. These effects are consequences of suppression of blood pressure and blood glucose, maintenance of pancreatic $\beta$-cell volume and normalization of cholesterol, phospholipids levels and triglyceride [3].

The cardioprotective effect of CUR has been evaluated by inhibition of the generation of free-radical and enhanced activities of detoxifying enzymes (glutathione-S transferase) in vivo [12]. The electrocardiographic and 
histopathological data from an animal model of cyclophosphamide-induced cardiotoxicity have been improved after 10 days of CUR administration in combination with piperine [12].

Extensive evidences have demonstrated CUR as a therapeutic and chemopreventive drug in cancer. A wide range of cancer cells, including liver, pancreatic, cervical, stomach, colon and breast cancer had their generation, transformation, proliferation and metastasis attenuated or suppressed by CUR [1]. The molecular mechanism of CUR in cancer seems to be related to the suppression of induction of early growth response- 1 gene product (EGR1), protein tyrosine kinase cascades and MAPK pathway [1]. CUR antiproliferative potential has been associated with traditional anticancer drugs such as paclitaxel and cyclophosphamide [13]. CUR could sensitive cells towards apoptosis and its modulatory role in expression of protein kinase $\mathrm{C}(\mathrm{PKC})$, nuclear factor- $\mathrm{\kappa} \mathrm{B}(\mathrm{NF}-\kappa \mathrm{B})$ and histone deacetylase (HDAC) in breast cancer has been investigated [13].

CUR antimicrobial properties have also been investigated, and the mechanism is related to the interaction between methoxy and hydroxyl groups from CUR and the prokaryotic cytoskeletal protein (FtsZ), a cell division initiating protein [14]. Furthermore, the yellowish appearance of CUR makes it a potential photosensitizer in photodynamic therapy of various diseases, such as infections and cancer $[5,15]$.

\section{Physicochemical properties}

CUR is also known as diferuloylmethane or (1E,6E)-1,7-bis(4-hydroxy-3-methoxyphenyl)-1,6-heptadiene-3,5dione [5]. Its structure $\left(\mathrm{C}_{21} \mathrm{H}_{20} \mathrm{O}_{6}\right.$; MW $\left.368.385 \mathrm{~g} / \mathrm{mol}\right)$ is composed of two aromatic rings with hydroxyl and methoxyl groups in the ortho position, which are connected by seven carbons with two $\alpha, \beta$-unsaturated carbonyl groups. It displays three possible forms, a $\beta$-diketonic and two isomers in an equilibrating keto-enol tautomeric form [16]. Between $\mathrm{pH} 3$ and 7, the enol form is more stable and predominant, due to the planar conformation. However, the conformation is dependent of solvent properties, temperature and polarity [17].

CUR is highly lipophilic, displays $\log$ P 3.29, and has demonstrated photosensitivity, low solubility in aqueous solutions and under alkaline conditions $(\mathrm{pH}>7)$, which makes them susceptible to degradation because of the formation of phenylate anion, increasing the production of CUR radicals [5,16].

Thus, the physicochemical properties of CUR are a challenge for the development of new drug-delivery systems. Since the high lipophilicity of CUR limits the bioavailability, the therapeutic efficacy and clinical applicability of CUR are hampered. Considering the Biopharmaceutical Classification System of drugs, CUR is classified as a class II drug, showing poor aqueous solubility but high permeability. A very low plasma concentration and no significant therapeutic activity are reported after the oral administration of CUR (8-12 g daily) [9]. Thus, there is a huge necessity for the development of carriers that can deliver CUR to the target site $[1,6,10,16]$.

\section{Micro/nanostructured systems containing CUR}

The development of carriers in order to solubilize or encapsulate CUR has grown really fast during the last decade. These carriers can improve solubility, stability, as well as precise and selective delivery of CUR to the target site and, consequently, improve the pharmacokinetics $[4,10]$. In an attempt to solve the physicochemical CUR drawbacks, many carriers have been developed such as micro/nanoparticles or capsules, micro/nanoemulsions, nanovesicles, lyotropic liquid vesicles and foams, among others.

Micro/nanoparticulate systems containing CUR have demonstrated successful improvement in wound healing, antioxidant, anti-inflammatory, tissue regenerating, as well as, Alzheimer's disease $[6,8,18]$. These formulations have been prepared using synthetic and natural polymers such as derivatives of cellulose, acrylic acid, lactic acid, glycolic acid, chitosan, gums, resins and gelatin, as well as lipid components such as stearic acid [4]. Therefore, the development of micro/nanoparticulate systems could improve the stability to light degradation, for example. In addition, it could provide biodegradability and biocompatibility, as well as high encapsulation efficiency and adequate surface potential [4-6]. Considering that CUR has demonstrated a wide range of pharmacological effects, some authors have developed particulate systems for an extensive variety of applications such as bladder [4], brain [11] as well as skin [6].

The colloidal systems called micro/nanoemulsions are used to encapsulate, solubilize and deliver hydrophobic drugs. Nanoemulsions display the property of thermodynamic stability, and they are transparent, while the microemulsions do not exhibit this property, and they are not transparent. During the development of emulsion-based systems, some parameters should be meticulously controlled, such as emulsion droplet size, oil composition, volume fraction, incorporation of CUR in the apolar phase and type of interfacial materials. These characteristics display huge importance, since they interfere in the stability, release and bioacessibility of the nano/microemulsions. Once 
the emulsion-based systems exhibit small droplet size, the drug could cross the intestinal lumen as well as the cutaneous barrier [5,16].

The spherical vesicles, comprised by at least one phospholipid bilayer, called liposomes, have been widely employed as CUR-delivery systems. These vesicles are able to carry hydrophilic and hydrophobic drugs, since they are structured in a polar core surrounded by an apolar region. In addition, liposomes containing CUR have been reported to present low toxicity, biodegradability and biocompatibility $[5,19]$.

Moreover, CUR has been incorporated in other drug-delivery systems, such as lyotropic liquid crystal systems, foams, micelles, hydrogels and nanofibers [19]. The lyotropic liquid crystal systems, classified as lamellar, cubic or hexagonal mesophases, can be administered in mucosal or topical delivery. The lipid-surfactant mesophases have the ability to absorb water from the physiologic liquids, thicken, and turn to gel phases in which the drugs could be released in a modified way [5]. The foams composed of porous silk fibroin scaffold have been loaded with CUR in order to provide quick healing of burns or wounds or to be employed as soft tissue replacements after postsurgical chemotherapy [19].

\section{Conclusion}

CUR is a promising molecule with a wide range of pharmacological effects, such as antimicrobial, anti-inflammatory, anticancer and wound healing, as well as effects in chronic diseases, such as Alzheimer's disease and diabetes. However, its physicochemical properties are not favorable to the development of new pharmaceutical forms due to the high hydrophobicity, light and $\mathrm{pH}$ instability that impair the absorption and bioavailability. Consequently, in the attempt to solve this problem, new strategies have been developed like micro/nanoencapsulated systems, vesicles and colloidal systems. Nevertheless, substantial gaps should be filled, since there is a lack of comparison between the performance of many formulations developed to deliver CUR in a specific aim, lack of toxicity and availability data of CUR in each drug-delivery system and lack of human and preclinical studies in order to evaluate the bioavailability. Therefore, only after these gaps are filled are we going to know if micro/nanoencapsulation is really the key for the improvement of the CUR bioavailability.

\section{Financial \& competing interests disclosure}

The authors have no relevant affiliations or financial involvement with any organization or entity with a financial interest in or financial conflict with the subject matter or materials discussed in the manuscript. This includes employment, consultancies, honoraria, stock ownership or options, expert testimony, grants or patents received or pending, or royalties.

No writing assistance was utilized in the production of this manuscript.

\section{References}

1 Khan S, Imran M, Butt TT et al. Curcumin based nanomedicines as efficient nanoplatform for treatment of cancer: new developments in reversing cancer drug resistance, rapid internalization, and improved anticancer efficacy. Trends Food Sci. Technol. 80, 8-22 (2018).

2 Rivera-Mancía S, Trujillo J, Chaverri JP. Utility of curcumin for the treatment of diabetes mellitus: evidence from preclinical and clinical studies. J. Nutr. Intermed. Metab. 14, 29-41 (2018).

3 Parsamanesh N, Moossavi M, Bahrami A, Butler AE, Sahebkar A. Therapeutic potential of curcumin in diabetic complications. Pharmacol. Res. 136, 181-193 (2018).

4 Oliveira MB, Da Silva JB, Montanha MC, Kimura E, Diniz A, Bruschi ML. Design and characterization of mucoadhesive gelatin-ethylcellulose microparticles for the delivery of curcumin to the bladder. Curr. Drug Deliv. 15(8), 1112-1122 (2018).

5 Alves RC, Fernandes RP, Fonseca-Santos B, Victorelli FD, Chorilli M. A critical review of the properties and analytical methods for the determination of curcumin in biological and pharmaceutical matrices. Crit. Rev. Anal. Chem. 25, 1-12 (2018).

6 Katas H, Wen CY, Siddique MI, Hussain Z, Hanini F, Fadhil M. Thermoresponsive curcumin/DsiRNA nanoparticle gels for the treatment of diabetic wounds: synthesis and drug release. Ther. Deliv. 8(3), 137-150 (2017).

7 Singh S, Khar A. Biological effects of curcumin and its role in cancer chemoprevention and therapy. Anti-Cancer Agents Med. Chem. 6, 259-270 (2006).

8 The Merck Index - An Encyclopedia of Chemicals, Drugs, and Biologicals. O’Neil MJ (Ed.). Royal Society of Chemistry, Cambridge, UK, 474 (2013).

9 Anand P, Kunnumakkara AB, Newman RA, Aggarwal BB. Bioavailability of curcumin: problems and promises. Mol. Pharm. 4(6), 807-818 (2007).

10 Akolade JO, Oloyede HOB, Salawu MO, Amuzat AO, Ganiyu AI, Onyenekwe PC. Influence of formulation parameters on encapsulation and release characteristics of curcumin loaded in chitosan-based drug delivery carriers. J. Drug Deliv. Sci. Technol. 45, 11-19 (2018) 
11 Doggui S, Dao L, Ramassamy C. Potential of drug-loaded nanoparticles for Alzheimers disease: diagnosis, prevention and treatment. Ther. Deliv. 3(9), 1025-1027 (2012).

12 Saeidinia A, Keihanian F, Butler AE, Bagheri RK, Atkin SL, Sahebkar A. Curcumin in heart failure: a choice for complementary therapy? Pharmacol. Res. 131, 112-119 (2018).

13 Roy M, Mukherjee S, Sarkar R, Biswas J. Curcumin sensitizes chemotherapeutic drugs via modulation of PKC, telomerase, NF- $\mathrm{B}$ and HDAC in breast cancer. Ther. Deliv. 2(10), 1275-1293 (2011).

14 Silva AC da, Santos PD de F, Silva JT do P, Leimann FV, Bracht L, Gonçalves OH. Impact of curcumin nanoformulation on its antimicrobial activity. Trends Food Sci. Technol. 72 (January 2017), 74-82 (2018).

15 Santezi C, Reina BD, Dovigo LN. Curcumin-mediated photodynamic therapy for the treatment of oral infections - a review. Photodiagnosis Photodyn. Ther. 21, 409-415 (2018).

16 Araiza-Calahorra A, Akhtar M, Sarkar A. Recent advances in emulsion-based delivery approaches for curcumin: from encapsulation to bioaccessibility. Trends Food Sci. Technol. 71, 155-169 (2018).

17 Jovanovic SV, Steenken S, Boone CW, Simic MG. H-atom transfer is a preferred antioxidant mechanism of curcumin. J. Am. Chem. Soc. 121(41), 9677-9681 (1999).

18 Khan AB, Mahamana R, Pal E. Review on mucoadhesive drug delivery system: novel approaches in modern era. Rajiv Gandhi Univ. Heal. Sci. J. Pharm. Sci. 4(4), 128-141 (2015).

19 Hussain Z, Thu HE, Amjad MW, Hussain F, Ahmed TA, Khan S. Exploring recent developments to improve antioxidant, anti-inflammatory and antimicrobial efficacy of curcumin: a review of new trends and future perspectives. Mater. Sci. Eng. C77, 1316-1326 (2017) 